\title{
Abnormal or Pathological* Leucocytes, as They Appear under the Copper Peroxidase Reaction.
}

\author{
63rd Report of the Peroxidase Reaction.
}

$\mathrm{By}$

\author{
Akira Sato, Tamotsu Suzuki and Ryoji Shibata. \\ (佐藤 彰) (鈴木 保) (柴田良次) \\ (From the Department of Pediatrics, Faculty of Medi- \\ cine, Tohoku Imperial University, Sendai. \\ Director: Prof. A. Sato.)
}

In the 50th Report ${ }^{15}$ of the Peroxidase Reaction we have discussed how different kinds of normal leucocytes-neutrophiles, eosinophiles, basophiles, monocytes and lymphocytes-would appear under the copper peroxidase reaction, and how they differed under the original copper peroxidase reaction of Sato and Sekiya ${ }^{2}$ and under its modification, the Tohoku-Pediatric Method.

Now we desire to discuss the difference of abnormal or pathological leucocytes under the two methods. As to the technique of these methods we shall refer to the 50th Report of the Peroxidase Reaction above mentioned.

A. Myelocytes.

1. Under the original Sato-Sekiya peroxidase stain they appear like blue rings each with one round or almost round nucleus intensely red-coloured. And the blue colour of the rings is so dark, that it may give one an impression that the blue can surpass that of normal neutrophiles in darkness probably due to narrowness of the light blue coloured perinuclear area. Thus even a beginner in hematology cannot mistake them for monocytes.

2. Under the Tohoku-Pediatric Method, myelocytes do not ap-

* Abnormal or pathological is not used in the strict sense; but here such blood leucocytes as will occur rarely or seldom in the physiological state are meant.

1) A. Sato, T. S u zuki and Ry. S hi bata, Tohoku J. Exp. Med., 1934, 24, 195.

2) A. S ato and S. Sekiya, Tohoku J. Exp. Med., 1926, 7, 111. 
pear any more like blue rings, but round cells with round, or almost round red nucleus. And the blue granules in the cytoplasma are rather coarse in contrast to the granules of monocytes, and even at the first glance the red nucleus appears to be distinctly interspersed with such coarse granules. Outlines of nucleus are sharper than under the original Sato-Sekiy a stain.

B. Myeloblasts (Cf. Fig. 1).

1. We shall relate of myeloblasts before promyelocytes. And again we shall discuss the Tohoku-Pediatric Method before the original S a to-S e kiy a stain, because under the former they present more characteristic features.

With the low power objective most peroxidase granules appear to have been drawn in most instance to one side or corner and remain collected there, and sometimes to both opposite sides. The characteristic pictures give one at one glance the impression that too many peroxidase-granules are on one side or in one corner (or in two opposite corners only), so that one can never forget the first impression once obtained. Of course there is no mistaking a myeloblaste for a monocyte with a round nucleus. Even excepting a striking difference between the intense red coloured nucleus with nucleoli of the myeloblast and the light red stained nucleus of the monocyte, peroxidase granules are very coarse and dense in the former, while in the latter they are rather fine and loose. Sometimes such blue needlelike formations as seen in eosinophiles are very often present in the myeloblast, so that then the cell appears to have grown a blue beard, while in a monocyte such formations are-so to speak-of a small scale, and if they occur, not frequent.

Though it is difficult for a beginner to recognize peroxidase-negative myeloblasts, yet in praxis it is not frequent to find this peroxidase-negative myeloblasts even in leukemic blood ; almost all myeloblasts are peroxidase-positive.

2. The appearance of myeloblasts under the original SatoSekiy a peroxidase stain is similar to that under the Tohoku-Pediatric Method with the paradox difference** that a myeloblast seems to be stained apparently darker with the Tohoku-Pediatric Method than with the original.

* The difference is of course not seen in such an illustration as Fig. 1 in the present paper, because myeloblasts stained under Sato-S ekiy a's stain appear naturally darker. But, if a film with this stain and another with the Tohoku-Pediatric Method are each examined for itself, then the paradox difference will be seen. 


\section{Promyelocytes (Cf. Fig. 2).}

Those who have studied typical myelocytes and typical myeloblasts under the original Sato-Sekiy a stain as well as the TohokuPediatric Method will recognize linking forms between myelocytes and myeloblasts with respect not only to the form of nucleus, but also to the distribution of peroxidase granules. The peroxidase granules are more numerous and are scattered throughout the whole cell, though a more or less strong propensity to keep them grouped in one corner or two, or even more, is still seen. In this respect there are all possible forms of transition. But thus far we have not seen a promyelocyte with such a blue beard as above mentioned in the item of the myeloblast. And promyelocytes seem like myeloblasts to be stained apparently darker under the Tohoku-Pediatric Method than under the original Sato-Sekiya stain.

D. Lymphoblasts (Cf. Fig. 3).

Lymphoblasts of course appear as red stained cells. They cannot be mistaken for peroxidase-negative myeloblasts which may occur in cases of myelogenous leukemia, because the colour of nucleus of a lymphoblast is noticeably darker than that of a myeloblast. Further, a lymphoblast has mostly a larger spaced cytoplasma than a myeloblast.

E. Plasma Cells and Türk's Forms (Cf. Fig. 4).

Under the original Sato-Sekiy a stain and under the TohokuPediatric Method they are very easily recognized, because they are the only cells with protoplasma stained in brownish-red with a shade of yellow, which is very characteristic of them. The perinuclear area is left unstained. Haematological beginners will easily differentiate them from lymphocytes and similar cells, even allowing for their morphological characteristics.

It is to be noted here that the granules and cytoplasma which are stained by the Gie m sa so characteristically basophile are stained in a distinguishing yellowish brown colour with safranin.

F. Normoblasts (Cf. Fig. 6).

It was stated in the 50th Report ${ }^{1)}$ of the Peroxidase Reaction that the Tohoku-Pediatric Method will take away all the red cells from the film with the exception of the nucleated red cells. Now the nucleus of a normoblast is very intensely red stained; much darker than that of a lymphocyte. While the protoplasma of the latter is stained faintly red, that of a normoblast is either not stained at all, or, if the protoplasma is polychromatic, yellowish stained, but never red-coloured. 


\section{Remarks (Cf. Figs. 5 and 6).}

1. The facility of differentiation with the use of the TohokuPediatric-Method will be shown in Fig. 5 and Fig. 6. Fig. 5 shows the Giemsa-stained picture of a blood specimen from a case of myeloblastic leukemia; one can easily see how difficult the differentiation of cell kinds is, while another specimen from the same case, when stained with the Tohoku-Pediatric-Method, a modification of the original Sato-Sekiya stain, will show cell kinds quite distinctly, even to a beginner.

2. As to the pathological eosinophiles and basophiles, the peroxidase granules of these will be understood easily if the 50th report ${ }^{13}$ on the peroxidase reaction is referred to. But this report is not sufficient, as far as the peroxidase granules of basophiles are concerned, of which a detailed description will be made in a later report..s)

\section{Summary.}

In the present paper it has been shown how abnormal or pathological leucocytes, peroxidase-negative as well as peroxidase-positive, and nucleated red cells will appear under the original copper peroxidase reaction of Sato-Sekiya and under the Tohoku-Pediatric Method, a modification of the original. And it has been shown how easily the abnormal leucocytes can be differentiated with these two methods, especially with the Tohoku-Pediatric Method.

3) Paper of T.Suzuki and M. Shinto, to be published in this Journal. 